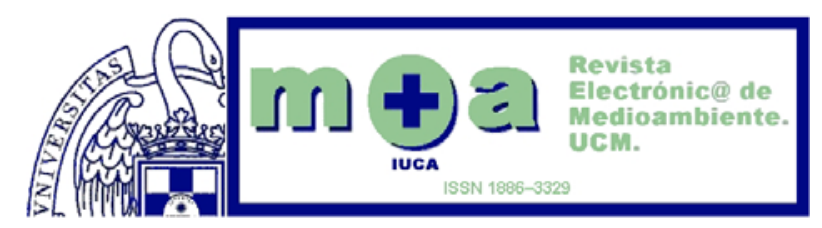

\title{
TRANSFORMACIONES DE LAS TENDENCIAS TURÍSTICAS Y SU INFLUENCIA EN EL MEDIO AMBIENTE: EL ESTUDIO DE CASO DE LA PROVINCIA DE ÁVILA
}

\author{
Fernando García Quiroga \\ Grupo de Investigación de la UCM: "Desarrollo y Gestión Ambiental del Territorio" \\ fgquiroga@ghis.ucm.com \\ María Sotelo Pérez \\ Grupo de Investigación de la UCM: "Desarrollo y Gestión Ambiental del Territorio" \\ maria_sotelo_perez@hotmail.com
}

Recibido: 12-01-2011

Aceptado: 09-05-2011

\section{RESUMEN}

En los últimos años los procesos de globalización de la economía, la política y la cultura tienen una creciente incidencia en los territorios y sociedades concretas (lo local). El desafío para las sociedades locales de tipo rural como las que encontramos en la provincia de Ávila, se está planteado en términos de insertarse en lo global de manera competitiva. En este sentido el avance del turismo rural en la última década dentro de la provincia de Ávila, va a ser un factor determinante, tanto en el medio ambiente de la provincia como en el desarrollo endógeno de la misma.

Palabras clave: turismo rural, desarrollo, medio ambiente, local.

\section{TRANSFORMATIONS ON TOURISM TRENDS AND THEIR IMPACT ON THE ENVIRONMENT: A CASE STUDY IN THE PROVINCE OF AVILA}

\begin{abstract}
In recent years the processes of globalization of the economy, politics and culture have a growing impact on specific territories and (local) societies. The challenge for rural-type local societies such as those found in the province of Avila, is cast in terms of inserting itself in a competitive manner in the wide global picture. From this point of view, the development of rural tourism in the last decade within the province of Ávila, will be a determining factor, both in the local environment as in the endogenous development of the province.
\end{abstract}

Keywords: rural tourism, development, environment, local. 


\section{TRANSFORMATIONS DES TENDANCES TOURISTIQUES ET LEUR INFLUENCE SUR L'ENVIRONNEMENT: UNE ÉTUDE DE CAS DANS LA PROVINCE DE AVILA}

\section{RESUMÉ:}

Au cours de ces dernières années, les processus de mondialisation de I'economie, la politique et la culture ont un impact croissant sur les territories et les societés (au niveau local). Le défi pour les sociétés ruraux locales, telles que celles trouvées dans la province de Avila, consiste à s'intégrer dans le global d' une manière compétitive. Dans ce sens, le progrès du tourisme rural au cours de la dernière décennie dans la province de Avila, sera un facteur determinante ayant une incidence sur I'environnement comme sur le développement endogène de la province.

Mots-clef: tourisme rural, développement, environnement, local.

\section{INTRODUCCIÓN Y ASPECTOS CONCEPTUALES}

El turismo se caracteriza por ser una actividad socioeconómica generalizada relativamente joven y puede englobar a un gran número de sectores económicos y ramas científicas como la geografía, la economía, la sociología, etc. Esto da lugar a una dificultad conceptual. Así, un geógrafo define el turismo basándose en el territorio, un economista se centrará en el consumo, un sociólogo se detendrá en los comportamientos y motivaciones turísticas, etc. En consecuencia no existe una única definición de turismo, todas ellas son correctas y contribuyen, de alguna manera a entender un poco mejor la complejidad inherente del mismo (Crosby, A. \& Moreda, A. 2002). Existen un sinfín de definiciones de turismo, a continuación se muestran algunas de ellas:

“El turismo es el conjunto de los fenómenos originados por los viajes...Estos se originan en un mercado, forman y engrosan las corriente turísticas y se dirigen a los núcleos receptores" (Fernández Fuster, L. 1985).

“El turismo es la suma de fenómenos y de relaciones que surgen de los viajes y de las estancias de los no residentes, en tanto en cuanto no están ligados a una residencia permanente ni a una actividad remunerada" (Hunziker, W \& Krapf, K. 1942).

"El turismo son los desplazamientos cortos y temporales de la gente hacia destinos de fuera del lugar de residencia y de trabajo, y las actividades emprendidas durante la estancia en esos destinos" (Burkart, A. J \& Medlik, S. 1981).

“El turismo es el movimiento temporal de la gente por periodos inferiores a un año, a destinos de fuera del lugar de residencia y de trabajo, las actividades emprendidas durante la estancia y las facilidades creadas para satisfacer las necesidades de los turistas" (Mathieson, A \& Wall. G. 1992).

Aunque todas estas definiciones se diferencian entre ellas por determinados elementos, también se desprenden algunas características comunes, como el 
desplazamiento físico, la estancia no permanente en el destino o la realización de actividades en dicho destino. De acuerdo con la definición de la OMT (Organización Mundial de Turismo), "el turismo comprende las actividades que realizan las personas durante sus viajes y estancias en lugares distintos al de su entorno habitual, por un periodo de tiempo consecutivo inferior a un año, con fines de ocio, por negocios y otros" (OMT. 1994).

No obstante, existe una serie de características que son propias del turismo. Por ejemplo, la actividad turística implica que una persona acomete un desplazamiento:

- Puede que este desplazamiento sea para menos de un día (excursionista/visitante).

- Puede ser un desplazamiento dentro de las fronteras de un país, dando así lugar a un viaje turístico interno.

- Podría ser un desplazamiento que cruzara una frontera, lo que se ubicaría en la clasificación de viaje turístico internacional.

El desarrollo del turismo de tipo rural suele ser algo deseable, debido a que rehabilita edificios que mantienen la arquitectura popular y suelen ser regentados por gente autóctona. El turismo rural es especialmente sensible de la conservación del entorno, pues "vive" de ello. Una manera de resolver el conflicto de intereses, entre el desarrollo del turismo y la conservación del medio ambiente, es transformar el territorio en un valor añadido y no solamente en un soporte físico. La información y la educación tanto de los profesionales dedicados al turismo como de los propios turistas, se convierte así en algo fundamental.

Las acciones relacionadas directamente con el turismo rural han acaparado buena parte de las ayudas de la iniciativa comunitaria Leader ("Liaisons entre activités de Developement de L'Economie Rural" - "Relaciones entre Actividades de Desarrollo de la Economía Rural"). Todo ello hace pensar que, en efecto, la función turística de los espacios rurales y naturales, sin ser la única alternativa al sector primario, sí se ha convertido en un elemento importante en ese nuevo abanico multifuncional al que dedica atenciones y cuidados la propia política de la Unión Europea (VV.AA. 2008).

“El turismo es una actividad dinámica y cambiante. Uno de los principales agentes de cambio son los empresarios que, mediante la combinación de perspicacia, creatividad y la confluencia de acontecimientos fortuitos están identificando, permanentemente oportunidades. Sin embargo, a pesar del papel central de los emprendedores en la actividad turística y en la evolución de los destinos turísticos" (Russell, R \& Faulkner, B. 2004) en concreto en el turismo rural, son muy pocos los trabajos de investigación al respecto que han analizado dicho papel.

El turismo rural, como cualquier otra estrategia de desarrollo, requiere de una serie de factores para sus éxitos, tales como la existencia de elementos de atracción (naturales y humanos), estrategias de promoción (comercialización de una comunidad y de sus atracciones turísticas a los potenciales turistas), infraestructuras turísticas, servicios y una actitud positiva de acogida por parte de los empleados de los negocios turísticos y de la comunidad en general. Asimismo, el componente empresarial y su papel en el fomento de los factores mencionados son igualmente importantes, porque de ello puede depender, en parte, el éxito del desarrollo turístico. Pero no hay que olvidar que el turismo rural es un producto de 
una comunidad y, como tal, necesita no sólo de las habilidades empresariales y de la presencia de empresas turísticas, sino también de las capacidades comunitarias locales (Pulido Fernández, Juan I. 2008).

El fomento del turismo rural es una de las claves, para avanzar en la implantación de un turismo sostenible y un desarrollo endógeno. La sostenibilidad del turismo se concibe como una vía hacia la gestión de todos los recursos, de forma que puedan satisfacer las necesidades económicas, sociales y estéticas, respetando al mismo tiempo la integridad cultural, los procesos ecológicos esenciales, la diversidad biológica y los sistemas que sostienen la vida. También está estrechamente relacionado con las iniciativas de implantación de la Agenda 21 Local. En este sentido la Ley 45/2007, del 13 de diciembre, para el desarrollo sostenible del medio rural, promueve el fomento del turismo rural a través de una adecuada ordenación de la oferta y la mejora de la demanda turística, con una atención preferente al turismo sostenible en las zonas rurales prioritarias y al agroturismo o turismo ligado a la actividad agraria.

Entre los motivos del éxito del turismo rural en nuestro país y sobre todo en el caso de la provincia de Ávila, se debe a varias causas, que interactúan entre ellas. La incorporación al mercado turístico de la población más joven, cuyos escasos recursos económicos lo impedían, que debido a la competitividad y variedad de precios que ofrece el turismo rural y a la propia configuración de este tipo de turismo, han optado mayoritariamente por él.

Entre las causas culturales o de evolución de la mentalidad colectiva, podemos citar la incuestionable crisis del modelo tradicional de sol y playa, donde el turista adopta una actitud pasiva. La nueva implantación de una nueva cultura del ocio, donde el descanso no se concibe como "no hacer nada", sino como un cambio de la actividad de la vida cotidiana. Los modelos existentes de turismo rural, responden perfectamente a esta nueva visión generalizada del concepto de ocio. Finalmente, no podemos obviar la difusión de las ideas de respeto al medio ambiente, que han contribuido sin duda a potenciar el turismo rural.

El turismo rural, ha servido en cierta manera, para convencer a muchos emprendedores dentro de la provincia de Ávila. El turismo como solución integral de los problemas de subdesarrollo no ha quedado en una exposición más o menos teoría, sino que se está plasmando en el incremento de los establecimientos que se dedican a ello. Como no podía ser de otra forma, el fenómeno del turismo rural, también está propiciando un intercambio más frecuente entre los habitantes del mundo rural y el urbano, lo que está contribuyendo a disminuir las importantes diferencias de mentalidad.

El desarrollo turístico actúa a menudo como catalizador económico y social para el cambio. El turismo es un negocio altamente segmentado, muy proclive a ofrecer oportunidades a empresarios de pequeña escala. Además el turismo es una actividad multisectorial que genera empleo directo (con trabajos creados específicamente ante la necesidad de abastecer y de servir a los turistas) y empleo indirecto (de tipo esporádico relacionado con el turismo que serán desviados a otros sectores de la economía que requieran esos servicios concretos).

"El turismo tiende a ser una actividad que requiere mucho empleo, debido a que el turismo es en esencia una actividad de prestación de servicios, tiende además a crear más empleos por unidad de inversión que otras actividades económicas" (Lickorish, J, L \& Jenkins, L. C. 1997). 
La conservación y mejora del medio ambiente, y su compatibilidad con un modelo de desarrollo socioeconómico del territorio, constituye indudablemente un factor estratégico tanto en términos de mejora de la competitividad de los productos y recursos turísticos locales, por ejemplo en lo que se refiere al aumento de los niveles de calidad de vida de la población. Por otro lado, el medio ambiente y el buen estado de conservación de los recursos naturales, constituye el capital fijo de cualquier iniciativa de turismo rural. Produciéndose la difícil relación entre la protección de los recursos naturales, y el incremento de la rentabilidad económica.

La sostenibilidad es básicamente un compromiso para superar las disfunciones ambientales que provoca el actual modelo económico y plantea un cambio de actitud dentro de un marco espacial y temporal concreto. Este marco espacial y temporal ha de estar limitado en un espacio de tiempo acorde con la sostenibilidad que presenta la propia tierra. El desarrollo turístico ha de plantearse una serie de medidas que ayuden a incrementar en general la calidad de los entornos donde se desarrolla la actividad turística, en este sentido se pueden plantear tres aspectos a tener en cuenta:

- Proporcionar al visitante experiencias enriquecedoras de calidad. Los turistas, cuando deciden pasar una estancia fuera de su domicilio habitual, mantienen una cierta expectativa de experimentar vivencias distintas a las de su vida cotidiana. Los responsables del destino han de plantear con imaginación y coherencia qué experiencias pueden desarrollarse según la vocación turística del lugar. En muchos casos, estas experiencias de calidad tienen una fuerte componente ambiental.

- Mantener, y en lo posible mejorar, la calidad del medio ambiente, tanto para el visitante como para la población local. Las deficiencias ambientales afectan muy negativamente a la imagen de las zonas turísticas.

- Mejorar la calidad de vida de la población local. La mejora de la calidad de vida de la población autóctona incrementa la seguridad de los destinos, reparte los beneficios de la actividad turística y fomenta la participación de la población residente en el desarrollo (Bosch Camprubí, R. et al. 1998).

\section{EVOLUCIÓN DEL TURISMO RURAL EN LA PROVINCIA DE ÁVILA}

En la actualidad, el turismo constituye la tercera actividad socioeconómica más importante de la UE, después de los sectores del comercio y la distribución, y la construcción. Si tenemos en cuenta los sectores relacionados con él, la contribución del turismo al producto interior bruto es todavía más importante; se estima que da lugar a más del $10 \%$ del PIB de la Unión Europea y que proporciona un $12 \%$ del empleo total. En relación con esto, si se observa la tendencia de los diez últimos años, el crecimiento del empleo en el sector turístico ha sido casi siempre más importante que en el resto de la economía. La Unión Europea sigue siendo el primer destino turístico del mundo, con 370 millones de llegadas de turistas internacionales durante el año 2008, lo que representa un $40 \%$ de las llegadas en todo el mundo. La competitividad del turismo está estrechamente relacionada con su sostenibilidad, ya que la calidad de los destinos turísticos depende mucho de su entorno natural y cultural y de su integración en una comunidad local. La sostenibilidad del turismo incluye muchos aspectos: la utilización responsable de los recursos naturales, la consideración del impacto medioambiental de las actividades (producción de residuos, presión sobre el agua, el suelo y la biodiversidad, etc.), la utilización de energías «limpias», la protección 
del patrimonio y la conservación de la integridad natural y cultural de los destinos, la calidad y la estabilidad de los puestos de trabajo creados, las repercusiones económicas locales o la calidad de la acogida [COM (2010) 352 final].

En España en el año 2010, se registró una llegada de 52,7 millones de turistas, algo más de un $1 \%$ de incremento sobre el año anterior. Esta cifra se aproxima a la media (53,7 millones) del periodo 2000-2010, la década que registró los incrementos más fuertes (MARM, 2010).

Por otra parte en 2010, el sector del turismo rural continuó la tendencia observada ya en los dos años anteriores, incrementándose el número de alojamientos y de plazas ofertadas, pero disminuyendo el número de turistas y de pernoctaciones. Los alojamientos rurales alcanzaron en España la cifra de 14.377, lo que supone un aumento del 3,5\%, mientras que las plazas llegaron a 126.222, casi un $4 \%$ más que el año anterior. Por su parte, la cifra de visitantes registrada fue de 2.666 .449 , (un $1,76 \%$ menos que en 2009) y la de pernoctaciones se situó en 7.674.431, acusando un descenso del 3,11\%. En general, la actividad del turismo rural se ha mantenido en 2010, pese a un contexto todavía adverso, especialmente para el turismo interior. No obstante, sí parece detectarse un desajuste entre el crecimiento de la oferta (alojamientos y plazas hoteleras) frente a la demanda (viajeros y pernoctaciones). Castilla y León es la Comunidad Autónoma que dispone de mayor infraestructura con 3.066 alojamientos, seguida a gran distancia de Cataluña (1.616), Asturias (1.339), Castilla-La Mancha (1.232) y Aragón (1.038). En su conjunto estas cinco Comunidades suman 9.615 establecimientos, el $67,1 \%$ del total. Por número de viajeros recibidos, también se sitúa en primer lugar Castilla y León, con más de medio millón de visitantes (596.843), seguido de Cataluña (298.878), Galicia (193.119), Cantabria (188.894) y Asturias (181.489). En el otro extremo se sitúan Canarias (49.772), Región de Murcia (32.277) y La Rioja (24.407) (MARM, 2010).

\section{Figura 1. Evolución del turismo rural en España en el periodo 2001-2010}

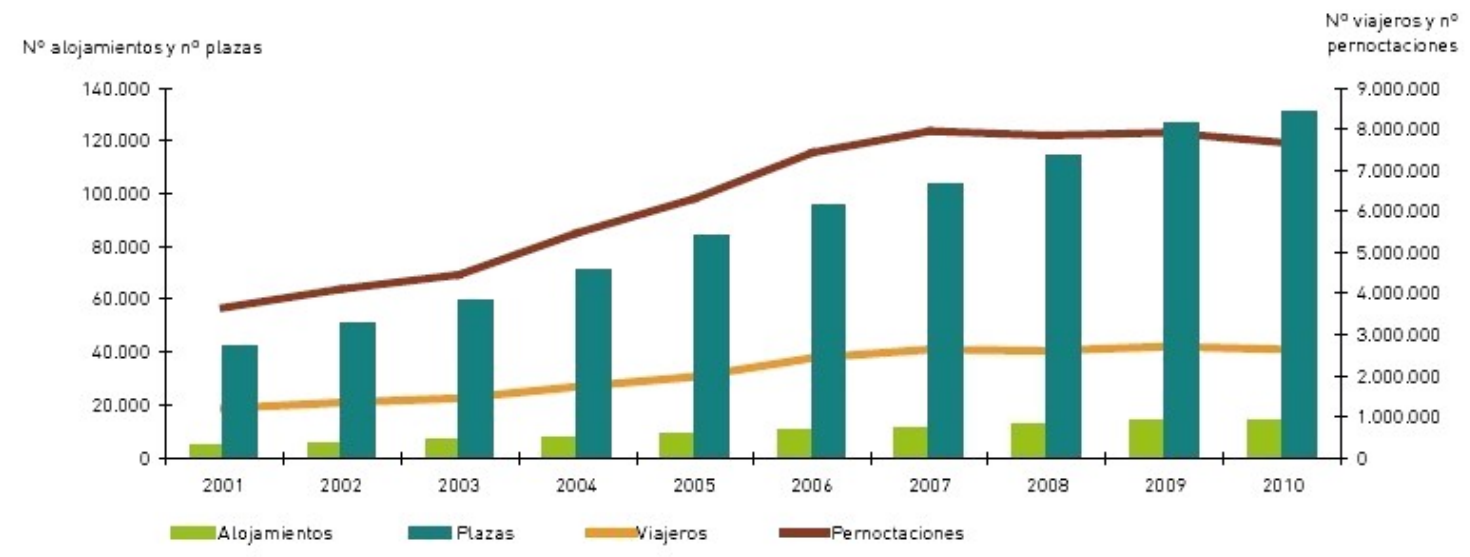

Fuente: MARM. Modificado 
Figura 2. Evolución de los establecimientos turísticos por tipo en el periodo 2000-2010

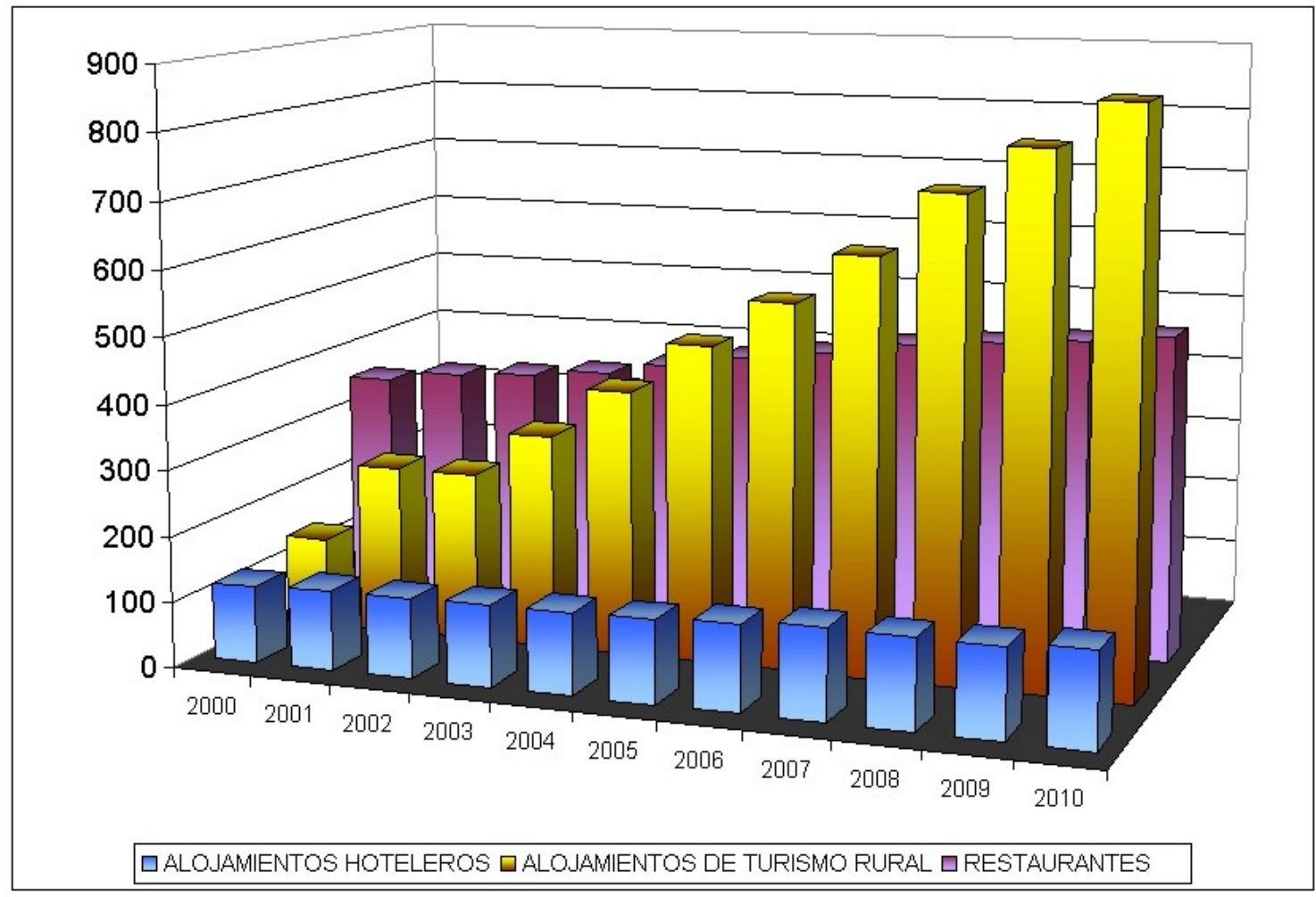

Fuente: J unta de Castilla y León. Elaboración propia

En este sentido, la provincia de Ávila ha desarrollando un sólido sector turístico basado en una potente hostelería y en los últimos años también con una oferta importante en turismo rural. En el gráfico sobre el número de establecimientos turísticos por tipos, entre el año 2000 al 2010, podemos ver la importancia gastronómica de Ávila, plasmada en el número de restaurantes de la provincia. Los restaurantes han tenido una progresión moderadamente ascendente, pasando de 349 en el año 2000, a 495 en el año 2010. La progresión en los alojamientos hoteleros, ha sido aún más moderada pasando de 116 en el año 2000, a 143 en el 2010. Este hecho se encuadra principalmente en que gran parte de los visitantes no siempre eligen pernoctar, debido a la cercaría a Madrid, y al desarrollo de gran cantidad de alojamientos de turismo rural, donde se ha pasado de 136 en el año 2000 hasta 862 en el año 2010, y todo esto a pesar de la crisis económica.

Esta circunstancia que tiene cada vez mayor relevancia dentro de los fenómenos turísticos de la provincia de Ávila, ya que puede que se trate del modelo turístico que presenta las características idóneas, para el desarrollo local de muchos municipios en declive socioeconómico. Podemos definir el turismo rural como "toda aquella actividad turística que tiene como escenario el espacio rural y cuyo desarrollo es respetuoso con el medio ambiente, además de garantizar la sostenibilidad de los recursos naturales" (Mesa Vila, M. 2000). 
El turismo rural en España, se originó en un principio como un fenómeno espontáneo, tanto en concepto de veraneo como, principalmente, de visitas de los emigrantes del éxodo rural a sus pueblos de origen. En ambos casos, hasta fechas muy recientes, no ha adquirido un carácter de actividad económica importante. El autentico auge por las iniciativas de turismo rural, se produce en 1991 a raíz de la aparición del Programa Comunitario LEADER, cuyo objetivo prioritario es incentivar actividades complementarias a la actividad agraria.

En el año 2000, el número de alojamientos destinados al turismo rural, era muy inferior y se repartían principalmente entre los términos municipales más cercanos a la Comunidad de Madrid. En el año 2010 esta cifra ha aumentado considerablemente, tanto en número de alojamientos como en su distribución por un mayor número de municipios por toda la provincia, si bien es cierto que donde tienen una mayor presencia es en los lugares más próximos a la Comunidad de Madrid, como sucedía en el año 2000.

\section{Mapa 1. Alojamientos de turismo rural por municipios (2000)}

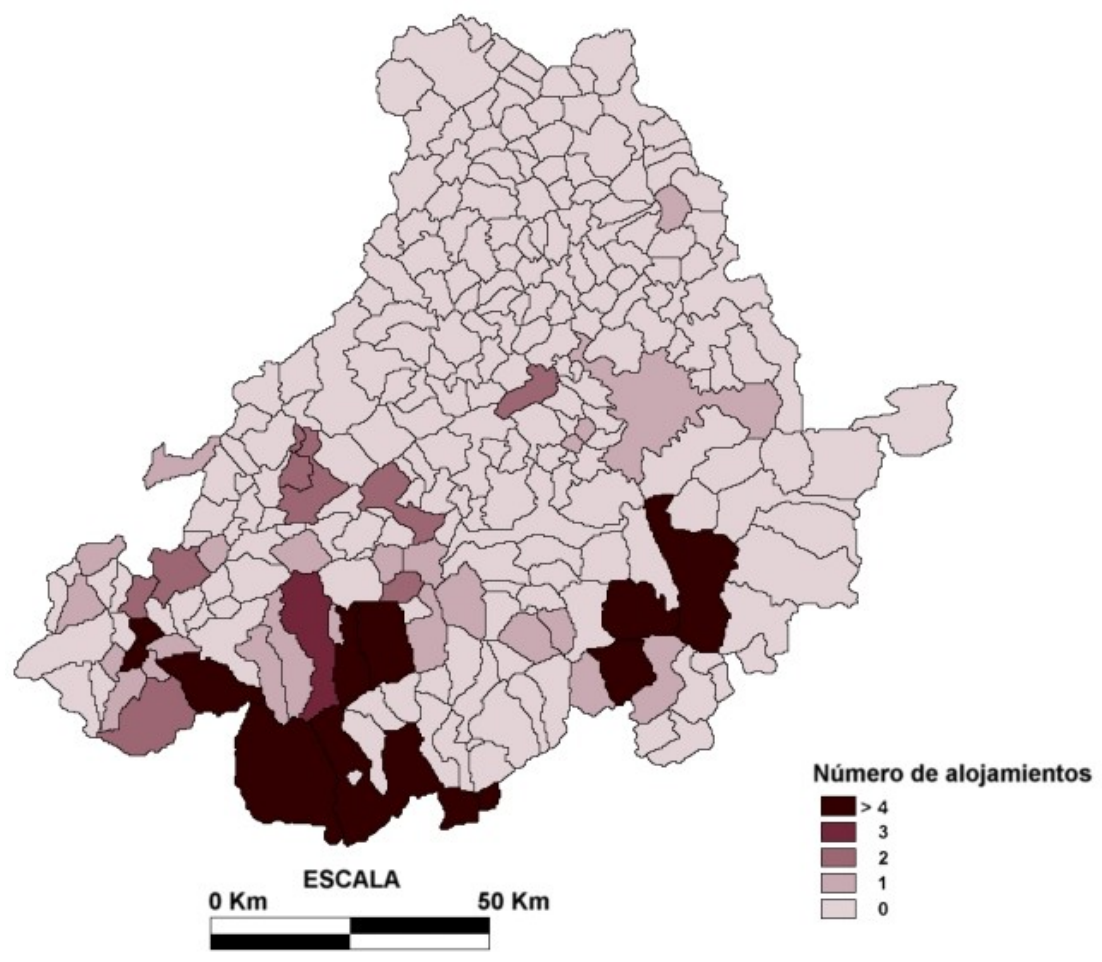

Fuente: J unta de Castilla y León. Elaboración propia 
Mapa 2. Alojamientos de turismo rural por municipios (2010)

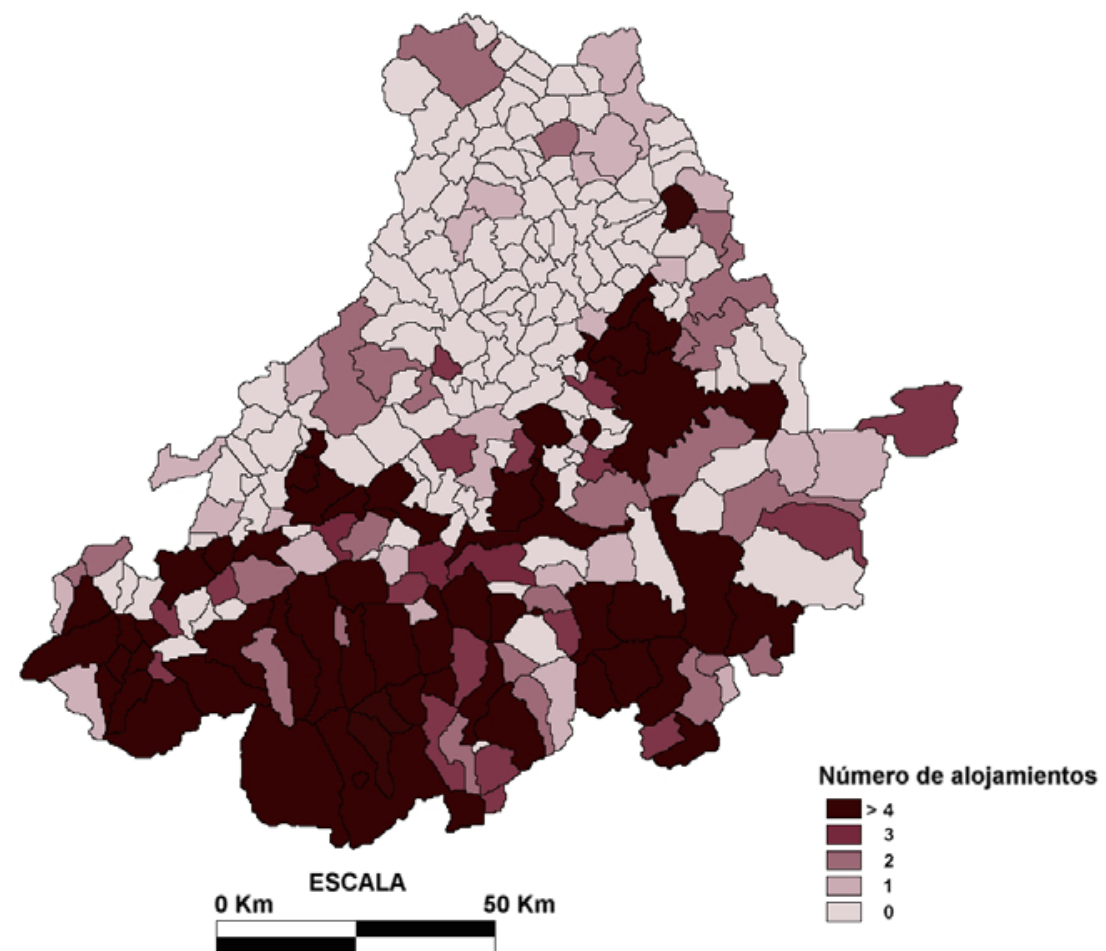

Fuente: J unta de Castilla y León. Elaboración propia

Mapa 3. Número de alojamientos de turismo rural en Castilla y León por provincias (2000)

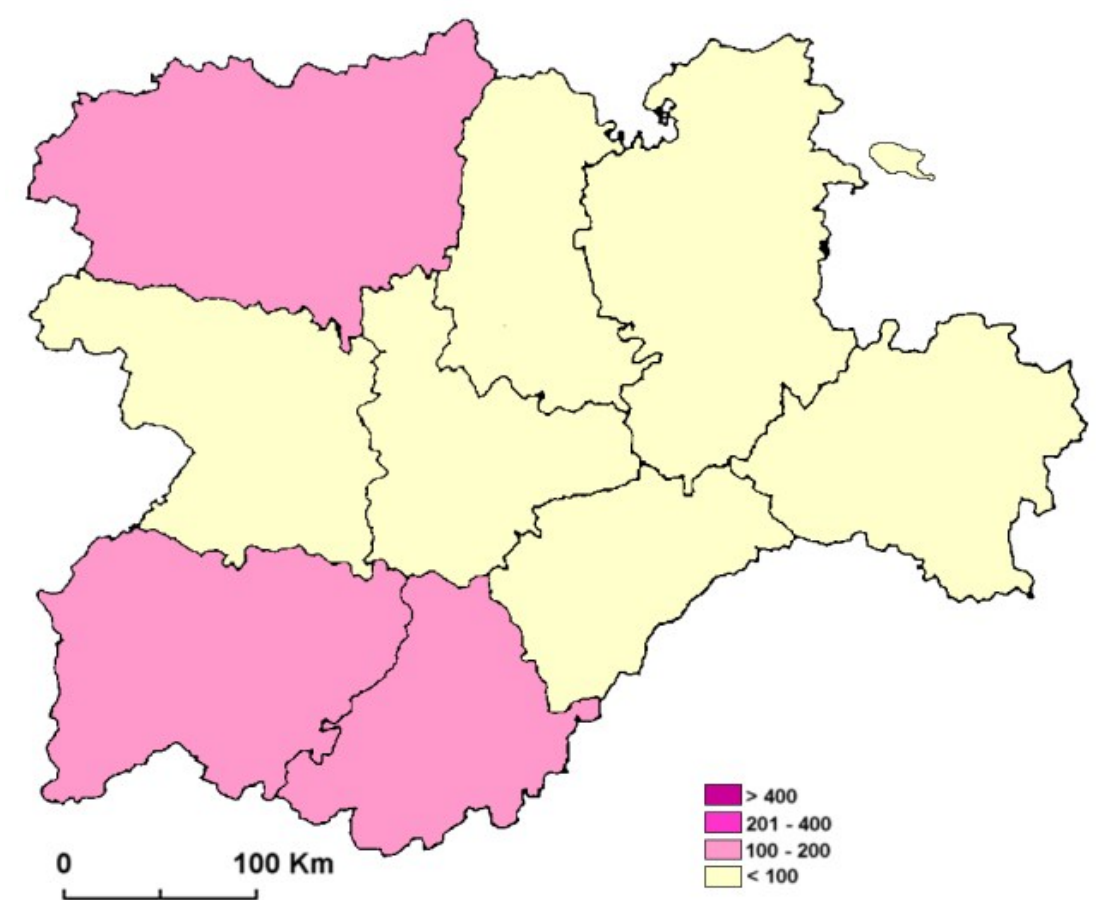

Fuente: J unta de Castilla y León. Elaboración propia 


\section{Mapa 4. Número de alojamientos de turismo rural en Castilla y León por provincias (2010)}

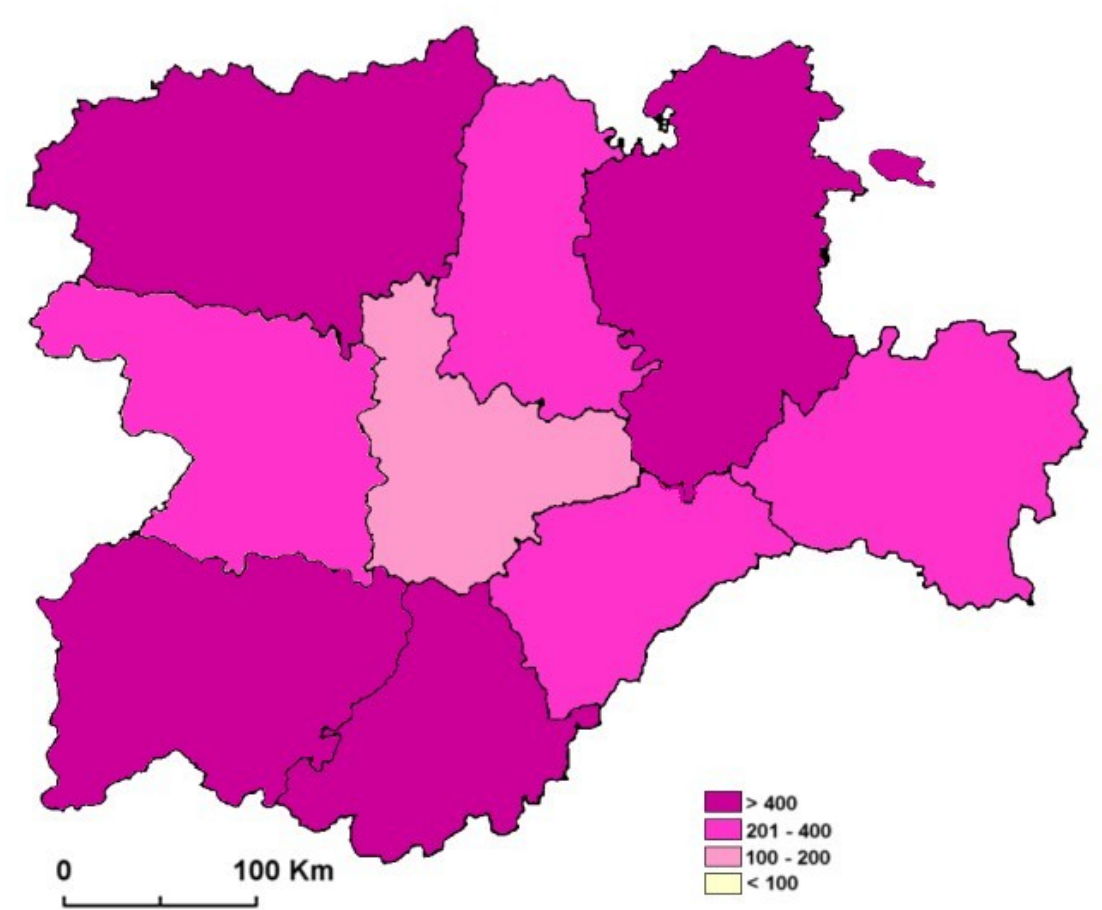

\section{Fuente: J unta de Castilla y León. Elaboración propia}

El fenómeno del aumento del turismo rural, es un fenómeno muy importante no sólo desde una perspectiva económica sino también social y ambiental, si observamos los dos mapas correspondientes a este mismo periodo, a nivel de toda la Comunidad Autónoma de Castilla y León, vemos algo muy similar, en el resto de la Comunidad Autónoma. En el año 2000, primer año del que se disponen datos estadísticos de este tipo de establecimientos dentro de la Junta de Castilla y León, el número total era de 784, mientras que en el 2010, el número ascendía a 3.628.

La conservación y mejora del medio ambiente, y su compatibilidad con un modelo de desarrollo socioeconómico del territorio, constituye indudablemente un factor estratégico tanto en términos de mejora de la competitividad de los productos y recursos turísticos locales, como en lo que se refiere al aumento de los niveles de calidad de vida de la población. Por otro lado, el medio ambiente y el buen estado de conservación de los recursos naturales, constituye el capital fijo de cualquier iniciativa de turismo rural. Produciéndose la difícil relación entre la protección de los recursos naturales y el incremento de la rentabilidad económica. 


\section{ANÁLISIS DE LAS INTERACCIONES DEL TURISMO RURAL Y SU INFLUENCIA EN EL MEDIO AMBIENTE DE LA PROVINCIA DE ÁVILA}

“La gente generalmente es racional, por lo que responde a estímulos: cuando resulta más costoso hacer algo, la gente tenderá a hacerlo menos; cuando resulta más fácil, económico o beneficioso, se inclinará a hacerlo con más frecuencia. Cuando sopesan sus opciones, las personas tienen presentes las limitaciones globales de las mismas: no sólo los costes y beneficios de una elección en particular, sino su presupuesto total. Además de considerar las consecuencias futuras de las elecciones presentes" (Harford, T, 2008). En este sentido, el hecho de un aumento de los establecimientos dedicados al turismo rural tanto en la provincia de Ávila, como en el resto de Castilla y León, no es un hecho fortuito, sino que obedece a una decisión económica racional. Por esta razón, e independientemente de los motivos personales que cada nuevo empresario dedicado al turismo rural, argumente para justificar su decisión de dedicarse económicamente a este tipo de turismo, debe de existir un ratio coste-beneficio favorable o perceptiblemente favorable. Esta afirmación se basa tanto en el aumento de los últimos años, como en la creación de nuevos establecimientos en plena crisis económica.

Un factor importante que se deriva del análisis del turismo rural abulense, es el hecho de que los términos municipales que han experimentado un mayor aumento de turismo rural, son precisamente donde mayor es su patrimonio natural, algo que puede traer externalidades negativas en forma de degradación o positivas si genera una concienciación tanto en la población autóctona como en el turista.

Una de las principales herramientas para la conservación y mantenimiento de la biodiversidad es la protección de los espacios naturales por parte de las Administraciones Públicas, lo que supone también la protección de las especies animales y vegetales que los habitan. De esta manera los Espacios Naturales Protegidos (ENP) constituyen uno de los instrumentos para la conservación de la naturaleza mejor contrastados, siempre y cuando exista una adecuada planificación y gestión de los mismos. Actualmente aunque existen disposiciones de declaración de espacios naturales protegidos más antiguas (caso de todos los existentes en la provincia de Ávila), la tipología de figuras de protección en España viene marcada por la Ley 42/2007 de 13 de diciembre del Patrimonio Natural y de la Biodiversidad que tiene en consideración las diferentes Directivas Europeas referentes a la conservación de la naturaleza (Aves o 79/409 CEE y Hábitats o 92/43/CEE). Esta Ley establece en su artículo 29 la clasificación de los ENP que en función de los bienes y valores a proteger, se clasifican en Parques, Reservas Naturales, Áreas Marinas Protegidas, Monumentos Naturales y Paisajes Protegidos.

Actualmente la lista de ENP, además de las figuras de protección recogidas en la legislación estatal y autonómica tienen su base en la Red Natura 2000, compuesta a partir de lo indicado en la ya citada Ley 42/2007, por los Lugares de Importancia Comunitaria ( LIC) que pasarán en un futuro a ser las Zonas Especiales de Conservación (ZEC), y por las Zonas de Especial Protección para las Aves (ZEPA). La Red Natura 2000 tiene la consideración de espacios protegidos, con el alcance y las limitaciones que las Comunidades Autónomas establezcan en su legislación y en los correspondientes instrumentos de planificación. En el caso de las ZEPA, estas son espacios adecuados en número y superficie para la conservación de las especies de aves incluidas en el Anexo IV de la Ley 42/2007 y para las aves migratorias de presencia regular en España, estableciéndose en ellas medidas para evitar las perturbaciones y de conservación especiales en cuanto a su hábitat, para garantizar su supervivencia y reproducción. 
Mapa 5. Ámbito territorial ocupado por los LICs en la provincia de Ávila

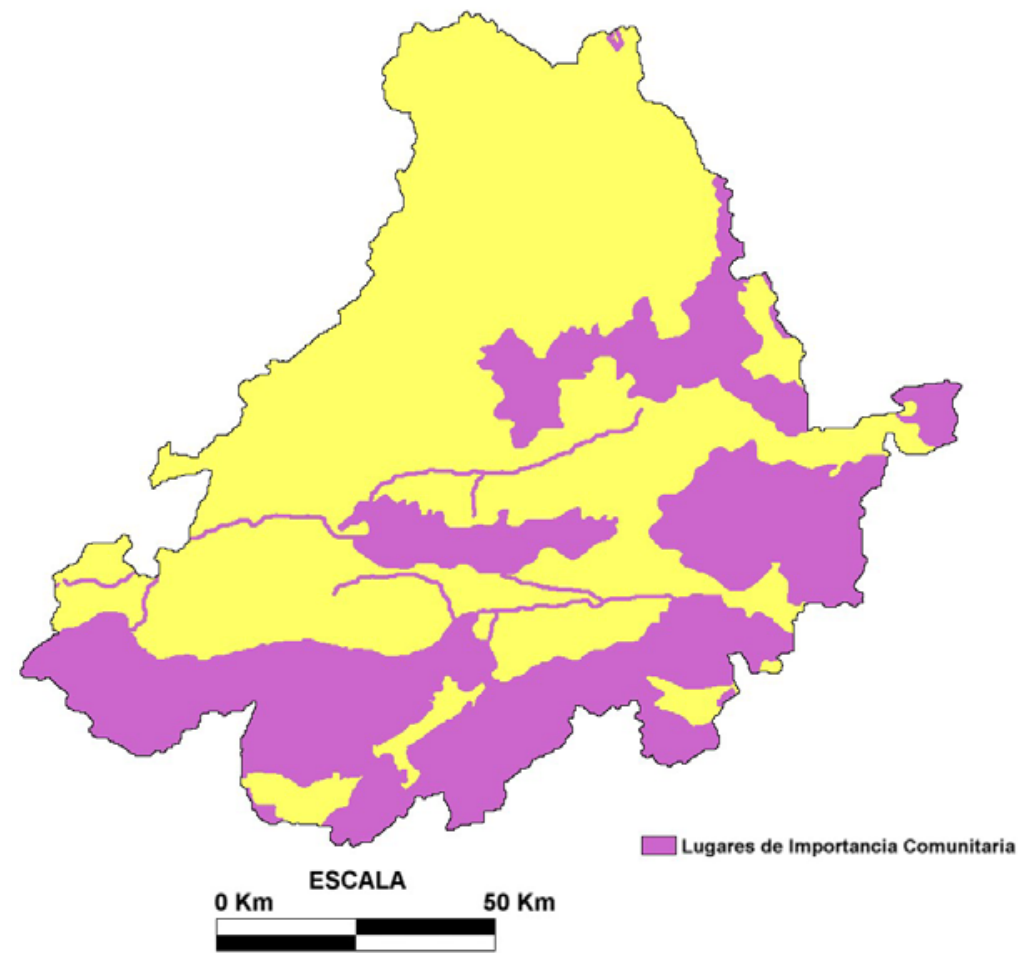

Fuente: J unta de Castilla y León. Modificado

Mapa 6. Ámbito territorial ocupado por las ZEPA en la provincia de Ávila

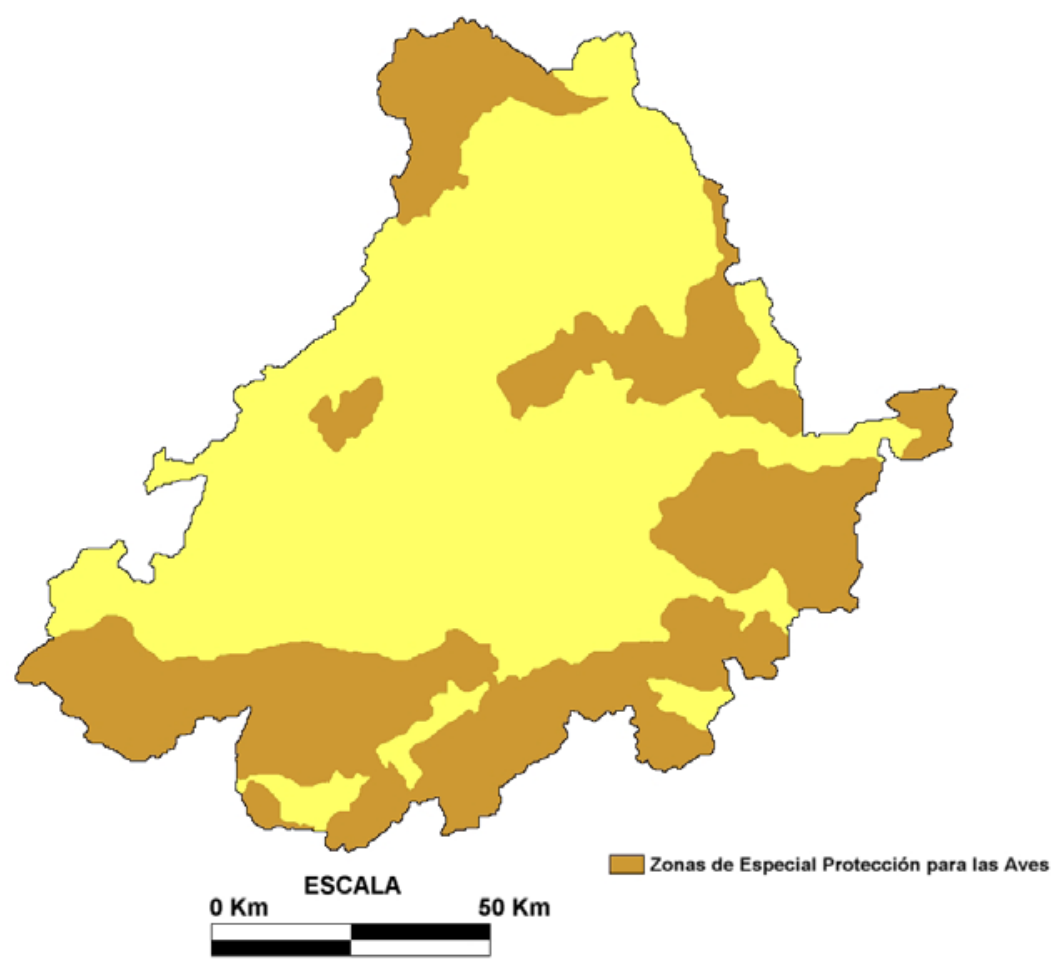

Fuente: Junta de Castilla y León. Modificado 
Para el caso de las especies de carácter migratorio que lleguen regularmente a territorio español, se deben tener en cuenta las necesidades de protección de sus áreas de reproducción, alimentación, muda, invernada y zonas de descanso, atribuyendo particular importancia a las zonas húmedas. El conjunto de la Red Natura supone la protección de una amplia extensión del territorio nacional, más de un $25 \%$. La provincia de Ávila, goza del privilegio de contar con un $37 \%$ de su superficie dentro de la Red Natura, el porcentaje más alto de todas las provincias de Castilla y León. En lo que respecta a los espacios con más endemismos corresponden básicamente a dos lugares, la Sierra de Gredos propiamente dicha y el Valle del Tiétar, siendo esto posible por el aislamiento y las diferencias de hábitats existentes a consecuencia de los desniveles existentes que determinan una amplio contraste entre la vegetación existente pasando de pinares, encinares y melojares en las partes bajas (éstos últimos por la elevada pluviometría) a prados y turberas en las cimas de las montañas. A pesar de ésta circunstancia físico natural, debe considerarse que el Valle del Tiétar es uno de los espacios más poblados y que más turismo recibe de la provincia por lo que las posibles amenazas para la conservación de la biodiversidad existente son mayores.

Al igual que en Gredos y el Tiétar otros municipios que atesoran más endemismos son aquellos situados en la Sierra de Villafranca, algunos de la Sierra de Ávila, y otros del extremo más oriental de la provincia como el municipio de Peguerinos. En éste caso la explicación de la presencia de tan elevado número de endemismos se debe de nuevo al hecho de recibir fauna tanto de especies presentes en la Sierra de Gredos como de aquellas existentes en la Sierra de Guadarrama, pues tienen en éste lugar el límite oriental y occidental de distribución. A ello además contribuye la existencia de unas masas forestales relativamente bien conservadas.

\section{Mapa 7. Distribución del número de vertebrados amenazados por municipios en la provincia de Ávila}

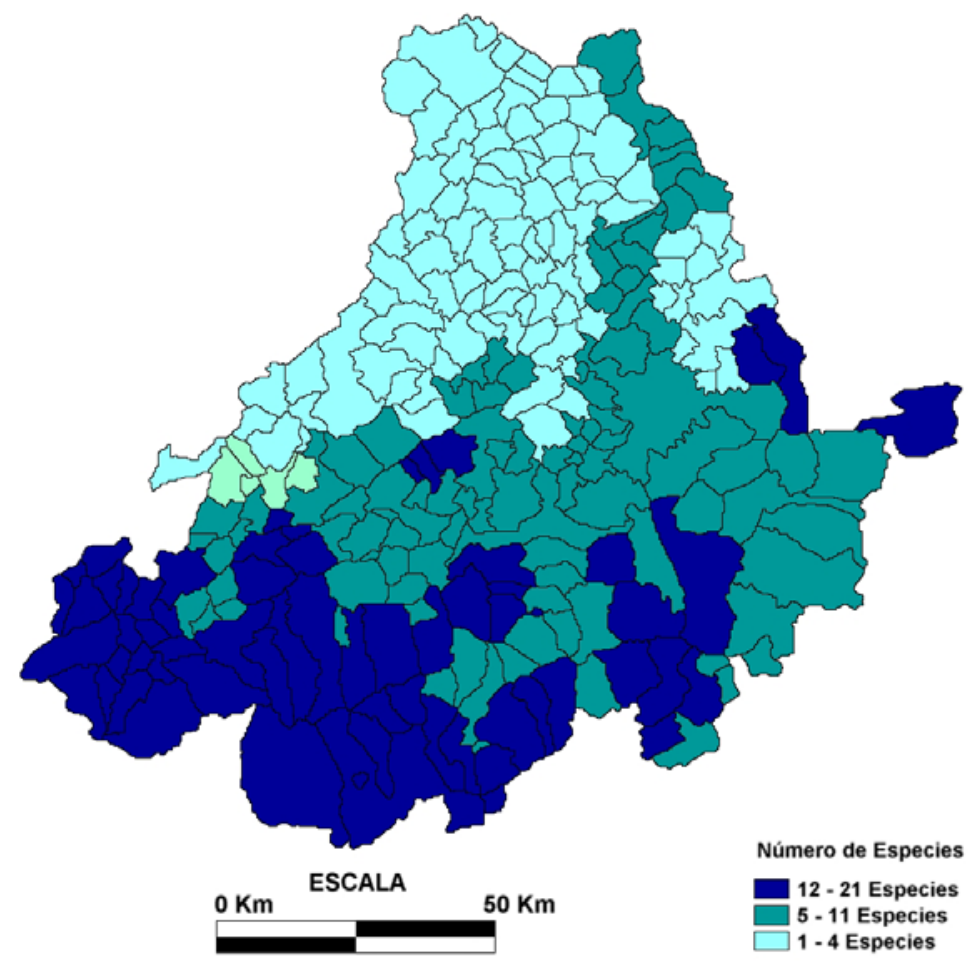

Fuente: MARM. Modificado 


\section{CONCLUSIONES}

Los territorios rurales siguen ostentando un papel fundamental en el contexto europeo. Más del $80 \%$ de la superficie de Europa tiene una condición rural y en ella se asientan un patrimonio natural y cultural de inconmensurable valor. Esas sí son, entre otras razones las que avalan el interés por preservar de un posible colapso, amplios territorios en toda Europa y explican la proliferación de incentivos y programas de desarrollo rural.

Por este motivo es necesario apostar por una verdadera estrategia de desarrollo que supere la consideración del medio rural como mero espacio de ocio estacional para satisfacer demandas de los espacios urbanos, y que contribuya realmente a mejorar la calidad de vida de los espacios rurales. Con esta finalidad, la integración de las nuevas dinámicas territoriales, sociales, económicas y ambientales que trae de la mano el turismo rural, debe hacerse acorde a los modelos históricos de organización del medio rural, y siguiendo los principios de la sostenibilidad ambiental.

Frente a otras actividades productivas - en este momento casi todas-, el turismo rural presenta una demanda expansiva que invita al optimismo y, generalmente menores exigencias de inversión para su desarrollo. Además, puede contribuir a la rehabilitación del patrimonio arquitectónico mediante la creación de establecimientos turísticos (museos, centros de visitantes, etc.) y a la preservación del paisaje como atractivo fundamental del espacio rural, favoreciendo los procesos de desarrollo endógeno, ya que involucra a la población local como máxima beneficiaria.

El turismo y, más concretamente el turismo rural, se muestra capaz de generar progreso en zonas atrasadas y regenerar áreas en declive. El avance del turismo rural durante los últimos años en la provincia de Ávila, es algo deseable debido a la labor social y ambiental que realiza. En este sentido, puede decirse que el turismo rural es el modelo turístico que presenta unas condiciones más favorables para el desarrollo local de los pequeños municipios abulenses, muchas veces pobres en capital humano, pero con gran riqueza en recursos medioambientales. La sostenibilidad del turismo se concibe como una vía hacia la gestión de todos los recursos, de forma que puedan satisfacer las necesidades económicas, sociales y estéticas, respetando al mismo tiempo la integridad cultural, los procesos ecológicos esenciales, la diversidad biológica y los sistemas que sostienen la vida.

La conservación y mejora del medio ambiente, y su compatibilidad con un modelo de desarrollo socioeconómico del territorio, constituye indudablemente un factor estratégico tanto en términos de mejora de la competitividad de los productos y recursos turísticos locales, como en lo que se refiere al aumento de los niveles de calidad de vida de la población. Por otro lado, el medio ambiente y el buen estado de conservación de los recursos naturales, constituyen el capital fijo de cualquier iniciativa de turismo rural, produciéndose la difícil relación entre la protección de los recursos naturales y el incremento de la rentabilidad económica.

El turismo rural, puede producir beneficios que se retroalimentan y muchas veces no se contabilizan, ya que mantienen paisajes naturales y urbanos al renovar las tradiciones de la arquitectura local, y respetar entornos de alto valor medioambiental ya que se les otorga el valor económico que producen a través de los turistas. En el más favorable de los casos, el turismo puede incluso ofrecer una manera de reanimar la vida social y cultural de la población local, dando así solidez 
a la comunidad residente, o al menos manteniendo población y favoreciendo las actividades locales.

Además la introducción de las tecnologías de información y la comunicación (TIC o IT "Information Technology"), es un gran aliado para el fomento del turismo en general y el turismo rural en particular, ya que ha servido entre otras muchas cosas para acercar la oferta a través de Internet, abaratando mucho la publicidad. Además ha contribuido en aumentar la capacidad de gestión, a través de las reservas "online".

\section{BIBLIOGRAFÍA}

Abad Soria, J \& García Quiroga, F (2009): La Huella Ecológica de la Provincia de Segovia. Instrumento para la Planificación y la Educación Ambiental. Caja Segovia obra social, Segovia.

BEECH, J \& ChADWICK, S (editores) (1995): Problemas específicos de gestión en las diversas empresas turísticas. Editorial Síntesis, Madrid.

BLANCO, A (editor) (2003): Castilla y León presente y futuro del turismo, Estudios de la Fundación Encuentro, Madrid.

BoERS, H. \& BosCH, M. (1995): La Tierra destino turístico. Introducción al turismo y al medio ambiente. SME/ Institute for Environmental Communicaction and Netherlands Institute of Tourism and Transport Studies.

Bosch Camprubí, R,. Puj ol Marco, L,. Serra Cabado, J \& Vallespino Riera, F (1998): Turismo y Medio Ambiente. Colección Ceura-Economía, Editorial Centro de Estudios Ramón Areces, Madrid.

BuRKART, A. J \& MEDLINK, S (1981): Tourism: past, present and future, Heinemann, London.

CALS, J., CAPELlà, J \& VAQuÉ, E (1995): El turismo en el desarrollo rural de España. Ministerio de Agricultura, Pesca y Alimentación, Madrid.

COM (2010) 352 final: “Europa, primer destino turístico del mundo: un nuevo marco político para el turismo europeo". Comunicado de la Comisión Europea al Parlamento Europeo, al Consejo, Al Comité Económico y Social Europeo http://ec. europa.eu/enterprise/sectors/tourism/files/communications/comm unication2010_es.pdf [Consultado el 20-05-2010].

Crosby, A \& MoredA, A (2002): Desarrollo y gestión del turismo en áreas rurales y naturales. Editado por Centro Europeo de Formación Ambiental y Turística, Madrid.

Crosby, A. et AL. (1993): El desarrollo turístico sostenible en el medio rural. Centro Europeo de Formación Ambiental y Turística, Madrid.

Esteban Alonso, A. DE (1998) “Los nuevos enfoques de la Sociología Medioambiental". En Editorial: Observatorio Medioambiental № 1. 
Esteban CuRiel, J, De (2007): La demanda del turismo cultural y su vinculación con el medio ambiente urbano: los casos de Madrid y Valencia. Tesis Doctoral.

FERNANDEZ Fuster, L (1985): Introducción a la teoría y técnica del turismo, Alianza Editorial, Madrid.

FLORENCIO CALDERÓN, A (2000): “Desarrollo local y desarrollo rural: el papel de los pequeños municipios. En Pérez Ramírez, B. y Carrillo Benito, E. Desarrollo local: Manual de uso. Federación Andaluza de Municipios y Provincias-ESIC, Madrid; pp. 557-599.

García QuiRoga, F (2006): "Bases y directrices para el desarrollo sostenible en el municipio de Vegas de Matute (Segovia)", Observatorio Medioambiental, Vol.9, pp. 267-296.

García Quiroga, F (2008): "Turismo, energía, transporte y medio ambiente en la provincia de Segovia", Observatorio Medioambiental, Vol. 11, pags. 149170.

García QuiRoga, F (2010): Desarrollo Endógeno y Turismo Rural en la Provincia de Segovia, Caja Segovia obra social, Segovia.

HARFORD, T. (2008): La lógica oculta de la vida. Editorial Temas de Hoy, S.A, Madrid, España.

HUNZIKER, W. \& KRAPF, K. (1942): Grundriss der Allgemeinen Fremdenverkhrslehre. Universidad de Berna, Suiza.

KANE, E. \& BRUn, M. (2001): Doing your Research. Marion Boyars, London.

LICKORISH, J, L \& JENKINS, L. C (1997): An Introduction to Tourism. Melbourne: Reed Educational and Professional Publishing.

MacCannelL, D (2003): El turista una nueva teoría de la clase ociosa, Editorial Melusina, Barcelona.

MARM (2011): Perfil Ambiental de España 2010. Informe basado en indicadores. Ministerio de Medio Ambiente y Medio Rural y Marino, Madrid.

MATHIESON, A \& WALL. G (1992): Tourism: economic, physical and social impacts, Longaman, London.

Mcl ntosh, R.W. \& Charles R. Goeldner. (1986): . Tourism: Principles, Practices and Philosophies. Toronto: John Wiley.

MesA Vila, M. (2000): “Desarrollo local y turismo: el turismo rural". En Pérez Ramírez, B. y Carrillo Benito, E. Desarrollo local: Manual de uso. Federación Andaluza de Municipios y Provincias-ESIC, Madrid; pp. 557-599.

MOTTIAR, Z \& RYAN, T (2007): "The Role of SMEs in Tourism Development: An Industrial District Approach Applied to Killarner, Ireland". En Thomas, R and Augustyn, M.: Tourism in the New Europe: Perspectivas on SME policies an practices. Elsevier, Amsterdam: 63-78. 
OMT (1994): Recomendaciones sobre estadísticas de turismo, Organización Mundial del Turismo, Madrid.

ORganizACIÓN MUNDIAL DEL TURISMO (2007): Datos Esenciales del Turismo Edición2007.http://unwto.org/facts/eng/pdf/highlights/UNWTO_Highlights07 _sp_HR.pdf [Consultado el 03-08-2008].

ORganización MUNDIAL DEL TURISMO (2008): UNWTO World Tourism Barometer June 2008. UNWTO. Consultado el 2008-08-01. Volumen 6 No. 2 [Consultado el 9-09-2008].

Page, Stephen J., Paul B, Graham B \& Jo C. (2001): Tourism: A Modern Synthesis. London: Thomson Learning.

PULIDO FERnÁNDEZ, JUAN I. (coord.) (2008): El turismo rural. Estructura económica y configuración territorial en España. Editorial Síntesis, Madrid.

Punch, K. (2003): Survey Research: the Basic. SAGE Publications Ltd., London.

RusSeLL, R \& FAULKNER, B (2004): “Entrepreneurship, chaos and the Tourism Area Lifecycle", Tourism Menagement, 31 (3): 556-579.

SHAW, G \& WilLiAMS, A. M (1989): “Torurism, Economic Development and the Role of Entrepreneurial Activity", en Cooper, C.P: Progress in tourism, Recreation and Hospitality Management, Belhaven Press, London: 67-81.

Sotelo, J. A. (2002): Desarrollo, Medio Ambiente, y Libertad en Europa, Oxford University Press, España.

Sotelo, J. A. (2007): Medio Ambiente, Desarrollo y Sostenibilidad, Modelos y políticas de actuación contradictorias, Oxford University Press, España.

VALLS, J (2004): Gestión de destinos turísticos sostenibles, Ediciones Gestión 2000, Barcelona.

VEAL, A. (1997): Research Methods for Leisure and Tourism a Practical Guide (2nd ed.) Pearson Education, Essex.

VV.AA. (2008): Turismo en espacios rurales y naturales, Atlas Nacional de España, IGN, Ministerio de Fomento, Madrid. 